12. Summary. The results of $\$ \S 2-7$ can be summarized in the following theorem:

THEOREM. The Kuratowski formula,

$$
\phi c \phi c \phi c \phi A=\phi c \phi A,
$$

is satisfied for $\phi$ equal to any of the operators $1, c, d, e, i, j, f, k$, and $s$.

Sections 10 and 11, together with this theorem, imply the following corollary:

Corollary. The equation

$$
(\phi c)^{\alpha+2} \phi A=(\phi c)^{\alpha} \phi A
$$

holds for every ordinal $\alpha$ equal to or greater than some finite or transfinite ordinal $\alpha_{0}$, and for $\phi$ equal to any of the operators $1, c, d$, $e, i, h, j, b, f, k$, and $s$.

UNIVERSITY OF IOWA

\title{
A CONDITION THAT A FIRST BOOLEAN FUNCTION VANISH WHENEVER A SECOND DOES NOT
}

\author{
BY J. C. C. MCKINSEY*
}

It is well known $\dagger$ that if two polynomials $f\left(x_{1}, \cdots, x_{n}\right)$ and $g\left(x_{1}, \cdots, x_{n}\right)$ in the field of complex numbers are such that $f$ vanishes whenever $g$ does not, then at least one of the two polynomials $f$ and $g$ is identically zero. The corresponding law, however, does not, in general, hold for Boolean functions, as may be seen by considering the two functions $x$ and $x^{\prime}$ in a twoelement Boolean algebra; the statement that either $x=0$ or else $x^{\prime}=0$ in a two-element Boolean algebra is, indeed, the familiar law of excluded middle. It is the purpose of the present note to determine the conditions on the coefficients of two Boolean functions in order that the first vanish whenever the second does not.

The condition found involves prime Boolean elements, which are defined as follows:

\footnotetext{
* Blumenthal Research Fellow.

$\dagger$ See, for example, Bocher, Introduction to Higher Algebra, p. 8.
} 
Definition: $A$ Boolean element $p \neq 0$ is called a prime if there exists no Boolean element $x$ such that $x \neq 0, x \neq p$, and $x<p$.

The condition, for functions of one variable, is given in the following theorem. It will be noticed that to say that neither $\alpha_{1} x+\alpha_{2} x^{\prime}$ nor $\beta_{1} x+\beta_{2} x^{\prime}$ vanishes identically is equivalent to saying that $\alpha_{1}+\alpha_{2} \neq 0$ and $\beta_{1}+\beta_{2} \neq 0$.

THEOREM 1. If $\alpha_{1} x+\alpha_{2} x^{\prime}$ and $\beta_{1} x+\beta_{2} x^{\prime}$ are two Boolean functions, neither of which vanishes identically, then a necessary and sufficient condition that, for every choice of $x$, either $\alpha_{1} x+\alpha_{2} x^{\prime}=0$ or else $\beta_{1} x+\beta_{2} x^{\prime}=0$ is that

$$
\begin{aligned}
\alpha_{1} \beta_{1}+\alpha_{2} \beta_{2} & =0 \\
\alpha_{1}+\beta_{1}+\alpha_{2}+\beta_{2} & =p, \text { a prime }:
\end{aligned}
$$

Proof: I first show that the condition is sufficient. By (2), we have $\alpha_{i}=0$ or $p$ for $i=1,2$, and $\beta_{i}=0$ or $p$, for $i=1,2$. By (1), we then have either $\alpha_{1}=0$ or else $\beta_{1}=0$, and either $\alpha_{2}=0$ or else $\beta_{2}=0$. Suppose $\alpha_{1}=0$, then, since $\alpha_{1}+\alpha_{2} \neq 0$, we have $\alpha_{2}=p \neq 0$, and hence $\beta_{2}=0$, and hence, since $\beta_{1}+\beta_{2} \neq 0$, we have $\beta_{1}=p$; thus our functions become $p x^{\prime}$, and $p x$. Suppose that, for some $y$, $p y^{\prime} \neq 0$, and $p y \neq 0$. Let $z=p y$; then $z=p y<p$, and $z=p y \neq 0$, also $z \neq p$ (since if $z=p$ we should have $0=p p^{\prime}=p z^{\prime}=p(p y)^{\prime}$ $\left.=p p^{\prime}+p y^{\prime}=p y^{\prime} \neq 0\right)$. Hence $p$ is not a prime, contrary to hypothesis; therefore, for every $x$, we have either $p x^{\prime}=0$ or else $p x=0$. The argument is similar if we assume $\beta_{1}=0$.

I now show that the condition is necessary. Suppose that neither $\alpha_{1} x+\alpha_{2} x^{\prime}$ nor $\beta_{1} x+\beta_{2} x^{\prime}$ vanishes identically, and that for every $x$ either $\alpha_{1} x+\alpha_{2} x^{\prime}=0$ or else $\beta_{1} x+\beta_{2} x^{\prime}=0$. Then for every $x$ we have $\left(\alpha_{1} x+\alpha_{2} x^{\prime}\right)\left(\beta_{1} x+\beta_{2} x^{\prime}\right)=\alpha_{1} \beta_{1} x+\alpha_{2} \beta_{2} x^{\prime}=0$, and hence $\alpha_{1} \beta_{1}+\alpha_{2} \beta_{2}=0$; therefore (1) holds. Moreover, the disjunction must hold, in particular, for $x=1,0$; hence we have either $\alpha_{1}=0$ or else $\beta_{1}=0$, and either $\alpha_{2}=0$ or else $\beta_{2}=0$. By hypothesis we cannot have $\alpha_{1}=0$ and $\alpha_{2}=0$, since this implies $\alpha_{1}+\alpha_{2}=0$; and similarly we cannot have $\beta_{1}=0$ and $\beta_{2}=0$. Hence either $\alpha_{1}=0$ and $\beta_{2}=0$, or else $\alpha_{2}=0$ and $\beta_{1}=0$. If $\alpha_{1}=\beta_{2}=0$, then we have, for every $x$, either $\alpha_{2} x^{\prime}=0$ or else $\beta_{1} x=0$; hence, in particular, $\alpha_{2} \alpha_{2}^{\prime \prime}=\alpha_{2}=0$, or $\beta_{1} x_{2}^{\prime}=0$; or, since $\alpha_{2} \neq 0$, we have $\beta_{1} \alpha_{2}^{\prime}=0$, which is equivalent to $\beta_{1}<\alpha_{2}$; similarly $\alpha_{2}<\beta_{1}$; it follows that $\alpha_{2}=\beta_{1}$. Thus we have, for every $x$, either $\alpha_{2} x=0$ or else $\alpha_{2} x^{\prime}=0$. 
If $\alpha_{2}$ is not a prime, then there is an element $c \neq 0, \alpha_{2}$ such that $c<\alpha_{2}$ (and hence such that $c \alpha_{2}^{\prime}=0$ ); then either $\alpha_{2} c=0$ or else $\alpha_{2} c^{\prime}=0$; but if $\alpha_{2} c=0$, then $0=\alpha_{2} c=\alpha_{2} c+\alpha_{2}^{\prime} c=c$, contrary to hypothesis, and if $\alpha_{2} c^{\prime}=0$, then $\alpha_{2} c^{\prime}+\alpha_{2}^{\prime} c=0$, so that $\alpha_{2}=c$, contrary to hypothesis. Hence $\alpha_{2}$ is a prime $p$, and we have $\alpha_{1}+\beta_{1}+\alpha_{2}+\beta_{2}=0+p+p+0=p$. If we suppose that $\alpha_{2}=\beta_{1}=0$, the same result follows. Thus the condition is necessary.

By an induction on the number of variables in $f\left(x_{1}, \cdots, x_{n}\right)$ and $g\left(x_{1}, \cdots, x_{n}\right)$, Theorem 1 can immediately be generalized to functions of $n$ variables, as follows:

Theorem 2. If $f\left(x_{1}, \cdots, x_{n}\right)$ and $g\left(x_{1}, \cdots, x_{n}\right)$ are two Boolean functions, neither of which vanishes identically, then a necessary and sufficient condition that, for every choice of $x_{1}, \cdots, x_{n}$, either $f\left(x_{1}, \cdots, x_{n}\right)=0$ or else $g\left(x_{1}, \cdots, x_{n}\right)=0$ is that

$$
\begin{aligned}
& \sum_{\alpha_{i, j}=0,1} f\left(\alpha_{1, j}, \cdots, \alpha_{n, j}\right) g\left(\alpha_{1, j}, \cdots, \alpha_{n, j}\right)=0, \\
& \sum_{\alpha_{i, j}=0,1}\left[f\left(\alpha_{1, j}, \cdots, \alpha_{n, j}\right)+g\left(\alpha_{1, j}, \cdots, \alpha_{n, j}\right)\right]=p \text {, a prime. }
\end{aligned}
$$

From this theorem we see that "primeless" Boolean algebras are the only Boolean algebras that possess (vacuously) the property that, if $f$ vanishes whenever $g$ does not, then either $f$ or $g$ is identically zero. It is known that primeless Boolean algebras exist.

We may also consider the statement that a given pair $f, g$ of Boolean functions, neither of which vanishes identically, are such that one vanishes whenever the other does not, as a generalized form of the law of excluded middle. Our theorem then asserts that to say a generalized form of the law of excluded middle holds in a Boolean algebra is equivalent to saying that the algebra is not primeless.

New York University 